\title{
Inteligencia emocional y bienestar psicológico en adolescentes limeños
}

\author{
Emotional intelligence and psychological well-being in adolescents from \\ Lima
}

Paula Solórzano-Gonzales ${ }^{1}$

\begin{abstract}
Resumen
Objetivo: determinar la relación entre inteligencia emocional y bienestar psicológico en adolescentes de un colegio parroquial mixto de Lima metropolitana. Materiales y métodos: estudio correlacional de corte transversal. Se realizó un censo a 233 adolescentes de 4to y 5to de secundaria en una Institución Educativa Parroquial de Lima Metropolitana. Se usaron como instrumentos el Inventario del cociente emocional BarOn (ICE), la Escala BIEPS-J y una ficha sociodemográfica. Para el análisis de los datos se utilizó el paquete estadístico STATA versión 14. Se empleó la prueba de Shapiro Wilk para determinar normalidad. Para las variables categóricas se usó la prueba de Chi-cuadrado y para numéricas la correlación de Pearson y Spearman. Se consideró una significancia estadística $\leq 0.05$. Resultados: no existe relación entre la inteligencia emocional y el bienestar psicológico $(\mathrm{p}=0.97)$. Se halló asociación entre la edad y la dimensión interpersonal $(\mathrm{p}=0.001)$ y entre la religión y la dimensión intrapersonal $(\mathrm{p}=0.01)$. El 50\% de participantes evidenció un nivel bajo de bienestar psicológico. Conclusión: de manera integral se observa que ambas asociaciones son considerados factores potenciales en el desarrollo de la inteligencia emocional, como adquirir habilidades sociales favorables, afianzar su autonomía, contar con herramientas necesarias para resolver conflictos y tomar decisiones .
\end{abstract}

Palabras clave: Inteligencia emocional; Salud del adolescente; Adolescencia; Religión (Fuente: DeCS).

\begin{abstract}
Objective: to determine the relationship between emotional intelligence and psychological well-being in adolescents of a mixed parochial school in metropolitan Lima. Materials and methods: cross-sectional correlational study. A census was made to 233 adolescents of 4th and 5th year of high school in a Parochial Educational Institution of Metropolitan Lima. The BarOn emotional quotient inventory (ICE), the BIEPS-J scale and a sociodemographic record were used as instruments. For the analysis of the data, the STATA version 14 statistical package was used. The Shapiro Wilk test was used to determine normality.. Chi-square was used for the categorical variables and the Pearson and Spearman correlation was used for numerical variables. Statistical significance was considered $\leq 0.05$. Results: there is no relationship between emotional intelligence and psychological well-being $(p=0.97)$. An association was found between age and interpersonal dimension $(\mathrm{p}=0.001)$ and between religion and the intrapersonal dimension $(\mathrm{p}=0.01)$. 50\% of participants evidenced a low level of psychological well-being. Conclusion: in an integral way it is observed that both associations are considered potential factors in the development of emotional intelligence, such as acquiring favorable social skills, strengthening their autonomy, having the necessary tools to resolve conflicts and make decisions .
\end{abstract}

Keys words: Emotional intelligence; Adolescent health; Adolescence; Religion (Source: DeCS).

Para citar:

Solórzano P. Inteligencia emocional y bienestar psicológico en adolescentes limeños . CASUS. 2019;4(1):30-36. ${ }^{1}$ Universidad Católica Sedes Sapientiae. Estudiante de Psicología.
Correo electrónico: paurora24.10@gmail.com
Fecha de recepción: 10-12-18

Fecha de envío a pares: $15-12-18$

Fecha de aprobación por pares: 08-03-19

Fecha de aceptación: 11-03-19 


\section{INTRODUCCIÓN}

A nivel nacional la población de adolescentes de 12 a 17 años es la segunda población más expuesta a situaciones de riesgo: la primera la constituyen los universitarios de 18 a 24 años $(1,2)$. Entre los adolescentes peruanos el $73.2 \%$ se encuentra más propenso al consumo de alcohol, el $46.1 \%$ consume tabaco, el $7.5 \%$ fuma marihuana, $2.2 \%$ usan PBC y $1.2 \%$ cocaína $(1,2)$. Paralelamente a la situación descrita se ha reportado un incremento de la violencia contra el adolescente en el Perú desde el año 2015. De este modo, el $45.4 \%$ de adolescentes fue víctima de violencia psicológica, el $24.1 \%$ de violencia física y el $19.4 \%$ de ambos tipos de violencia (3-5). Del mismo modo, el Ministerio de Salud refiere que en las instituciones educativas un $43.4 \%$ de adolescentes varones y un $32.4 \%$ de mujeres fue agredido físicamente al menos una vez en su vida por parte de sus mismos compañeros (6). Así mismo, en el contexto educativo se reportan entre los adolescentes problemas de interacción social, familiar, personal, académico, entre otros (7-9). Estos elementos afectan el bienestar psicológico pues impactan la realización y satisfacción con la vida desde un estado de salud emocional, física y psicológica (8).

En esta etapa de vida se va afianzando la personalidad. La misma, se ve influenciada por el autoconcepto conformado por la impresión consciente que tiene de sí mismo basándose en sus vivencias con los demás y las nuevas conductas que él mismo se atribuye (9). Por estos motivos es importante el desarrollo adecuado de sus relaciones interpersonales comprendidas en la dinámica de su grupo social $(10,11)$.

Por su parte, en las distintas situaciones de riesgo o contextos de interacción social, se pueden ver afectado el control emocional, las relaciones consigo mismo y con los demás emergiendo la importancia de una adecuada inteligencia emocional. Esta comprende el reconocimiento de los propios sentimientos y el de los demás, para su manejo adecuado en una dinámica interrelacional que el adolescente sostiene con los otros y consigo mismo (12). Por otro lado, este término
Tabla 1. Descripción de las variables principales y sociodemográficas

\begin{tabular}{|c|c|c|}
\hline & $\mathbf{n}$ & $(\%)$ \\
\hline \multicolumn{3}{|l|}{ Sexo } \\
\hline Masculino & 117 & 50.87 \\
\hline Femenino & 113 & 49.13 \\
\hline Edad $(m \pm D E)$ & \multicolumn{2}{|c|}{$15.48 \pm 0.56$} \\
\hline \multicolumn{3}{|l|}{ Lugar de nacimiento } \\
\hline Lima & 216 & 93.91 \\
\hline Provincia & 3 & 1.30 \\
\hline Extranjero & 11 & 4.78 \\
\hline \multicolumn{3}{|l|}{ Religión } \\
\hline Católica & 186 & 80.87 \\
\hline Cristiana & 24 & 10.43 \\
\hline Otra religión & 3 & 7.39 \\
\hline Ninguna religión & 17 & 7.39 \\
\hline \multicolumn{3}{|l|}{ Tipo de familia } \\
\hline Nuclear & 144 & 62.61 \\
\hline Extensa & 50 & 21.74 \\
\hline Reconstituida & 9 & 3.91 \\
\hline Monoparental & 27 & 11.74 \\
\hline \multicolumn{3}{|l|}{ Uso de tiempo libre } \\
\hline Deportes & 77 & 33.48 \\
\hline Lectura & 38 & 16.52 \\
\hline Estudios & 38 & 16.52 \\
\hline Desarrollo de un arte & 40 & 17.39 \\
\hline Trabajo & 8 & 3.48 \\
\hline Tareas domésticas & 29 & 12.61 \\
\hline \multicolumn{3}{|l|}{$\begin{array}{l}\text { Estado civil de los } \\
\text { padres }\end{array}$} \\
\hline Solteros & 34 & 14.78 \\
\hline Casados & 140 & 60.87 \\
\hline Viudos & 8 & 3.48 \\
\hline Divorciados & 18 & 7.83 \\
\hline Convivientes & 30 & 13.04 \\
\hline \multicolumn{3}{|l|}{$\begin{array}{l}\text { Inteligencia emocional } \\
(m \pm D E)\end{array}$} \\
\hline Intrapersonal & \multicolumn{2}{|c|}{$109.48 \pm 10.8$} \\
\hline Interpretación & \multicolumn{2}{|c|}{$74.41 \pm 9.22$} \\
\hline Adaptabilidad & \multicolumn{2}{|c|}{$94.54 \pm 7.05$} \\
\hline Manejo de estrés & \multicolumn{2}{|c|}{$81.16 \pm 11.46$} \\
\hline Animo general & \multicolumn{2}{|c|}{$84.55 \pm 7.19$} \\
\hline Impresión & \multicolumn{2}{|c|}{$110.73 \pm 10.86$} \\
\hline C.E. Total & \multicolumn{2}{|c|}{$157.41 \pm 9.17$} \\
\hline \multicolumn{3}{|l|}{ Bienestar psicológico } \\
\hline Bajo & 96 & 41.74 \\
\hline Promedio & 105 & 45.65 \\
\hline Alto & 29 & 12.61 \\
\hline
\end{tabular}

engloba habilidades muy distintas, aunque complementarias a la inteligencia exclusivamente cognitiva $(12,13)$. La inteligencia emocional en los adolescentes se encuentra en evolución si se afecta llega a repercutir en el bienestar psicológico (14). De tal forma, el bienestar psicológico es un 
Tabla 2. Asociación de bienestar psicológico e inteligencia emocional

\begin{tabular}{lcccc}
\hline $\begin{array}{l}\text { Inteligencia } \\
\text { Emocional }\end{array}$ & Bajo & Medio & Alto & p-value \\
\hline C.E. Total & $158.14 \pm 8.16$ & $156.40 \pm 9.51$ & $158.62 \pm 10.94$ & 0.488 \\
Dimensiones & media $\pm \mathbf{D S}$ & media \pm DS & media $\pm \mathbf{D S}$ & \\
$\quad$ Intrapersonal & $110.19 \pm 10.05$ & $109.28 \pm 11.34$ & $107.89 \pm 11.36$ & 0.364 \\
$\quad$ Interpretación & $73.77 \pm 8.28$ & $74.43 \pm 9.43$ & $76.45 \pm 11.22$ & 0.198 \\
$\quad$ Adaptabilidad & $94.89 \pm 6.83$ & $93.76 \pm 7.23$ & $96.24 \pm 6.92$ & 0.159 \\
$\quad$ Manejo de estrés & $82.24 \pm 11.89$ & $80.08 \pm 10.99$ & $81.58 \pm 11.79$ & 0.437 \\
$\quad$ Animo general & $85.10 \pm 6.55$ & $83.94 \pm 7.18$ & $84.96 \pm 9.10$ & 0.774 \\
Impresión & $110.31 \pm 10.89$ & $111.28 \pm 10.66$ & $110.14 \pm 11.75$ & 0.674 \\
\hline
\end{tabular}

concepto amplio que incluye dimensiones sociales, subjetivas y psicológicas, así como comportamientos relacionados con la salud en general que llevan a las personas a funcionar de un modo positivo. Con lo anterior se entiende un buen autoconcepto, relaciones positivas con los demás, el tener un propósito de vida y el adecuado manejo frente al entorno (15).

Teniendo en cuenta que la inteligencia emocional y el bienestar psicológico podrían ser considerados como moduladores del comportamiento de los adolescentes en la interacción familiar, el grupo de amigos y la comunidad resulta importante conocer la relación entre el nivel de inteligencia emocional y del bienestar psicológico en un grupo de adolescentes escolares de secundaria en un colegio parroquial mixto de Lima Metropolitana.

\section{MATERIALES Y MÉTODOS}

Estudio de tipo correlacional de corte transversal con enfoque cuantitativo (16). Se realizó un censo a 233 adolescentes de 4to y 5to de secundaria de una institución educativa parroquial de Lima Metropolitana. Las variables principales fueron: la inteligencia emocional (IE) que comprende el reconocimiento de los propios sentimientos y el de los demás, para su manejo adecuado en una dinámica interrelacional que el adolescente sostiene con los otros y consigo mismo (12), asimismo, es una variable cuantitativa que se midió mediante el Inventario de inteligencia emocional ICE de BarOn estandarizado previamente en una muestra de 3.375 niños $y$ adolescentes limeños de 7 a 18 años hallándose coeficientes de validez entre 0.77 y 0.88 (7). El inventario contiene siete dimensiones: intrapersonal, interpersonal, adaptabilidad, manejo de estrés, ánimo general e impresión (9).

El bienestar psicológico (BP) incluye dimensiones sociales, subjetivas y psicológicas, así como comportamientos relacionados con la salud en general que llevan a las personas a funcionar de un modo positivo (15), variable categórica politómica ordinal que fue medida a través de la Escala BIEPS-J, comprendido tres niveles: bienestar bajo (29 a 32), bienestar medio (33 a 36) y bienestar alto (37 a 39) (11, 17).

En relación a las variables sociodemográficas se consideraron la edad: variable cuantitativa discreta; el sexo: variable categórica dicotómica (masculino y femenino); lugar de nacimiento: variable categórica politómica (Lima, Provincia y Extranjero); religión: variable categórica politómica (Católica, evangélica, Otra religión, Ninguna Religión); tipo de familia: variable categórica politómica (Nuclear, Extensa, Reconstruida, Monoparental); uso de tiempo libre: variable categórica politómica (Deporte, Lectura, Estudio, Desarrollo de un arte, Trabajo, Tareas domésticas) y estado civil de los padres: variable categórica politómica (Solteros, Casados, Viudos, Divorciados, Convivientes).

Para el análisis de los datos se utilizó el paquete estadístico de STATA 14. Se empleó la prueba de normalidad Shapiro Wilk para las variables IE y edad. Para las variables categóricas se hizo uso la prueba de Chi-cuadrado; además, para las variables numéricas se empleó la Correlación de Pearson y la Correlación de Spearman. Por otro lado, en la correlación de variables numéricas y 
Tabla 3. Asociación de inteligencia emocional y de bienestar psicológico con las variables sociodemográficas

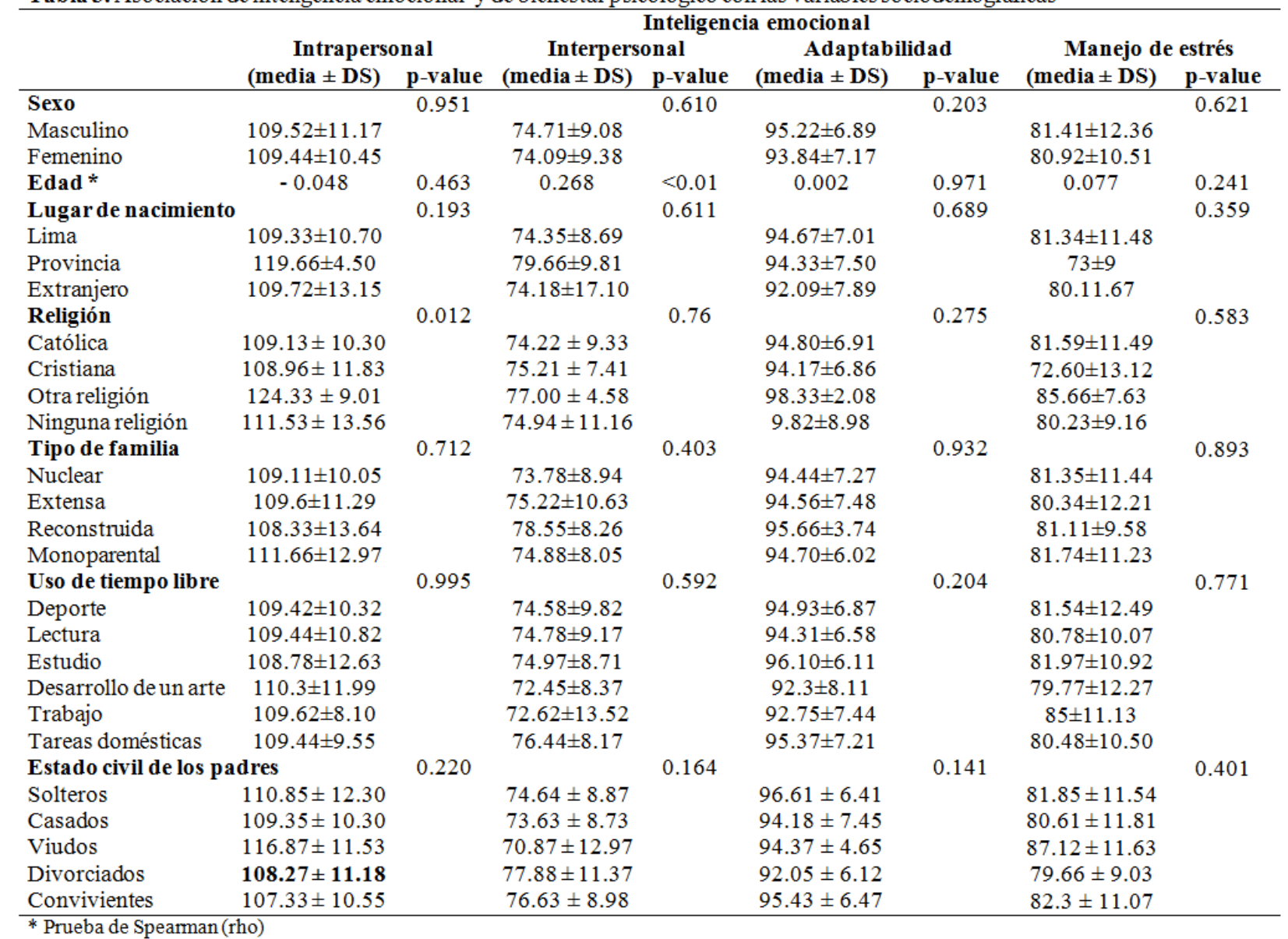

categóricas se utilizaron las pruebas U de Mann Whitney, Kruskal-Wallis, ANOVA. Se consideró un nivel de significancia estadística $\leq 0.05$.

El estudio siguió las directrices bioéticas en la investigación con seres humanos y se protegió la información proveniente de los encuestados con su anonimización.

\section{RESULTADOS}

En la tabla 1 se muestra que de los 230 alumnos encuestados el $50.87 \%$ es de sexo masculino. El 93.91\% nació en Lima, el 80,87\% de religión Católica y el $33.48 \%$ dedicó su tiempo libre a la ejecución de actividades deportivas. Así mismo, el $62.61 \%$ pertenecía a una familia nuclear, el $60.87 \%$ de sus padres estaban casados. El $41.74 \%$ evidenció un nivel bajo de bienestar psicológico al momento de la encuesta.

En la tabla 2 se observa que no se halló relación significativa entre la inteligencia emocional y el bienestar psicológico $(\mathrm{p}=0.48)$. Sin embargo, como se observa en la tabla 3 , existe una relación significativa entre la edad y la dimensión interpersonal de la inteligencia emocional $(\mathrm{p}=0.001)$. También se observó una asociación significativa entre la religión y la dimensión intrapersonal de la variable inteligencia emocional $(\mathrm{p}=0.012)$.

\section{DISCUSIÓN}

El estudio reveló que no existe relación significativa entre la inteligencia emocional y el bienestar psicológico. Sin embargo, existe asociación entre la edad y la dimensión interpersonal de inteligencia emocional, así como entre la religión y la dimensión intrapersonal de la misma. Por otra parte, se halló que una parte importante de la muestra presentó un nivel bajo de bienestar psicológico.

Se observó que la inteligencia emocional y el bienestar psicológico en los estudiantes encuestados no están asociados significativamente. 
Tabla 3. continúa

\begin{tabular}{|c|c|c|c|c|c|c|c|c|}
\hline \multicolumn{5}{|c|}{ Inteligencia emocional } & \multicolumn{4}{|c|}{ Bienestar psicológico } \\
\hline & \multicolumn{2}{|c|}{ Animo general } & \multicolumn{2}{|c|}{ Impresión } & \multirow{2}{*}{$\begin{array}{c}\text { Baja } \\
\text { (media } \pm \text { DS) }\end{array}$} & \multirow{2}{*}{$\begin{array}{c}\text { Moderada } \\
\text { (media } \pm \text { DS) }\end{array}$} & \multirow{2}{*}{$\begin{array}{c}\text { Alta } \\
(\text { media } \pm \text { DS) }\end{array}$} & \multirow{2}{*}{$\begin{array}{c}\text { P- } \\
\text { value }\end{array}$} \\
\hline & $($ media \pm DS $)$ & p-value & $($ media \pm DS $)$ & p-value & & & & \\
\hline Sexo & & 0.925 & & 0.230 & & & & 0.656 \\
\hline Masculino & $84.48 \pm 6.38$ & & $109.88 \pm 0.91$ & & $47(40.17)$ & $53(45.30)$ & $17(14.53)$ & \\
\hline Femenino & $84.62 \pm 7.96$ & & $111.61 \pm 1.10$ & & $49(43.36)$ & $52(46.02)$ & $12(10.62)$ & \\
\hline Edad * & -0.114 & 0.082 & 0.107 & 0.103 & $15.47(0.59)$ & $15.49(0.56)$ & $15.52(0.51)$ & 0.753 \\
\hline Lugar de nacimiento & & 0.269 & & 0.716 & & & & 0.826 \\
\hline Lima & $84.50 \pm 7.22$ & & $110.61 \pm 10.85$ & & $89(41.20)$ & $99(45.83)$ & $28(12.96)$ & \\
\hline Provincia & $80 \pm 7$ & & $115.33 \pm 2.88$ & & $1(33.33)$ & $2(66.67)$ & 0 & \\
\hline Extranjero & $86.81 \pm 6.30$ & & $111.81 \pm 12.72$ & & $6(54.55)$ & $4(36.36)$ & $1(9.09)$ & \\
\hline Religión & & 0.920 & & 0.634 & & & & 0.882 \\
\hline Católica & $84.52 \pm 7.25$ & & $157.58 \pm 9.13$ & & $79(42.47)$ & $83(44.62)$ & $24(12.90)$ & \\
\hline Cristiana & $85.60 \pm 7.51$ & & $156.69 \pm 10.71$ & & $8(34.78)$ & $11(47.83)$ & $4(17.39)$ & \\
\hline Otra religión & $82.66 \pm 10.78$ & & $162.66 \pm 4.50$ & & $2(66.67)$ & $1(33.33)$ & 0 & \\
\hline Ninguna religión & $83.82 \pm 6.05$ & & $156.11 \pm 8.16$ & & $7(41.18)$ & $9(52.94)$ & $1(5.88)$ & \\
\hline Tipo de familia & & 0.928 & & 0.669 & & & & 0.194 \\
\hline Nuclear & $84.55 \pm 6.87$ & & $110.90 \pm 11.04$ & & $63(43.75)$ & $65(45.14)$ & $16(11.11)$ & \\
\hline Extensa & $84.22 \pm 8.28$ & & $111.22 \pm 9.76$ & & $14(28.00)$ & $28(56.00)$ & $8(16.00)$ & \\
\hline Reconstruida & $84.44 \pm 6.94$ & & $112.33 \pm 12.26$ & & $3(33.33)$ & $4(44.44)$ & $2(22.22)$ & \\
\hline Monoparental & $85.22 \pm 7.12$ & & $108.40 \pm 11.63$ & & $16(59.26)$ & $8(29.63)$ & $3(11.11)$ & \\
\hline Uso de tiempo libre & & 0.355 & & 0.696 & & & & 0.795 \\
\hline Deporte & $84.16 \pm 5.84$ & & $110.55 \pm 10.26$ & & $34(44.16)$ & $30(38.96)$ & $13(16.88)$ & \\
\hline Lectura & $85.73 \pm 6.07$ & & $111.89 \pm 11.38$ & & $15(39.47)$ & $20(52.63)$ & $3(7.89)$ & \\
\hline Estudio & $84.07 \pm 6.73$ & & $110.13 \pm 9.83$ & & $17(44.74)$ & $17(44.74)$ & $4(10.53)$ & \\
\hline Desarrollo de un arte & $83.47 \pm 8.85$ & & $110.115 \pm 12.48$ & & $15(37.50)$ & $20(50.00)$ & $5(12.50)$ & \\
\hline Trabajo & $81.25 \pm 873$ & & $106 \pm 11.90$ & & $5(62.50)$ & $3(37.50)$ & 0 & \\
\hline Tareas domésticas & $87.06 \pm 8.88$ & & $112.58 \pm 10.12$ & & $10(34.48)$ & $15(51.72)$ & $4(13.79)$ & \\
\hline Estado civil de los pac & dres & 0.572 & & 0.397 & & & & 0.162 \\
\hline Solteros & $86.26 \pm 6.97$ & & $111.88 \pm 10.50$ & & $19(55.88)$ & $12(35.29)$ & $3(8.82)$ & \\
\hline Casados & $84.11 \pm 7.82$ & & $111.37 \pm 10.80$ & & $56(40.00)$ & $66(47.14)$ & $18(12.86)$ & \\
\hline Viudos & $84.12 \pm 4.42$ & & $111.12 \pm 12.92$ & & $2(25.00)$ & $3(37.50)$ & $3(37.50)$ & \\
\hline Divorciados & $85.88 \pm 5.59$ & & $107.16 \pm 13.10$ & & $4(22.22)$ & $11(61.11)$ & $3(16.67)$ & \\
\hline Convivientes & $84 \pm 5.52$ & & $108.5 \pm 9.46$ & & $15(50.00)$ & $13(43.33)$ & $2(6.67)$ & \\
\hline
\end{tabular}

Contrariamente a lo hallado, en otro estudio (18) reporta significancia en la asociación entre la inteligencia emocional, el bienestar psicológico y la estabilidad emocional. La diferencia en los resultados puede responder a la disimilitud entre las poblaciones de ambos estudios, tanto en edad como en aspectos socioculturales. En este sentido, los participantes están en una etapa donde se presentan conflictos tanto individuales como a nivel social, como presión del grupo $(1,4)$, rebeldía, frustración $(4,8)$ o la experimentación en el consumo de sustancias psicoactivas $(11,19)$.

Por otro lado, se observó que la edad se asocia significativamente con la dimensión interpersonal de inteligencia emocional. Dicho resultado es corroborado con otra investigación que sostiene que en la adolescencia el área interpersonal se ve potencializada pues el vínculo con los padres pierde importancia cobrando valor las relaciones con sus compañeros de escuela y otros grupos (20). Lo anterior, debido a la necesidad de pertenencia a un grupo, de consolidación y definición de su identidad, de la búsqueda de autonomía y del reconocimiento de otras personas (21)

De igual manera, se halló relación entre la religión y la dimensión intrapersonal de la inteligencia emocional. Tal resultado es reportado en otra investigación, la cual menciona que la espiritualidad, autorreflexión e interiorización en los adolescentes se desarrolla con mayor facilidad en aquellos que han tenido una crianza cultivada en valores y creencias religiosas (22). El aspecto religioso denota un favorable desarrollo personal y social, en vista de que el joven puede adquirir un mayor autocontrol, autoconocimiento, autoestima y manejo de la frustración, entre otros (22).

Por otra parte, un porcentaje considerable de la muestra obtuvo un nivel bajo de BP. Este resultado se contrapone al de otro estudio uruguayo (19) que hace referencia a un nivel alto de esta variable en 
adolescentes de secundaria, debido a que contaban con una adecuada visión del futuro, un proyecto de vida, y un buen nivel de autoaceptación. Los resultados del presente estudio pueden deberse a factores contextuales. En este sentido, en Lima los adolescentes encuentran propensos al consumo de alcohol, el tabaco y al uso de sustancias estupefacientes $(1,2)$. Paralelamente, se ha reportado violencia contra el adolescente, donde un $45.4 \%$ fue víctima de violencia psicológica, el $24.1 \%$ de violencia física y el $19.4 \%$ de ambos tipos de violencia (3-5).

Es necesario mencionar para la mejor comprensión de los hallazgos como limitación que con el diseño propuesto no es posible verificar el comportamiento de las variables a lo largo del tiempo. Igualmente, que las respuestas de pruebas psicológicas utilizadas pueden haber sido influenciadas por el sesgo de deseabilidad social.

Asimismo, resulta necesario obtener información de otras variables sociodemográficas que pudieron enriquecer los presentes hallazgos y modular las variables objeto de estudio, como la presencia de enfermedad, consumo de drogas, y las características de la relación con los padres. Entre las fortalezas se encuentra el uso de cuestionarios estandarizados para medir los constructos objeto de estudio. Por otro lado, se trabajó con una muestra amplia y representativa de la población estudiantil de colegios parroquiales.

\section{CONCLUSIONES}

El estudio reveló que cuatro de cada diez adolescentes encuestados presentaron un nivel bajo de bienestar psicológico. Aunque los hallazgos señalan que no existe relación significativa entre la inteligencia emocional y el bienestar psicológico. Sin embargo, existe asociación entre la edad y la dimensión interpersonal de inteligencia emocional, así como entre la religión y la dimensión intrapersonal de la misma. En este sentido, resaltan la importancia de las relaciones interpersonales en esta etapa de la vida y el aporte de las creencias religiosas al desarrollo de la inteligencia emocional. Por último, estos hallazgos señalan la necesidad de diseñar programas de intervención psicológica que promuevan el desarrollo de la inteligencia emocional en beneficio del bienestar psicológico en adolescentes peruanos.

\section{REFERENCIAS BIBLIOGRÁFICAS}

1. Martínez, A. Chavez., V. Tapia, L. Vertiz, R. Epidemiología de drogas en población urbana peruana: encuesta en hogares Perú: CEDRO. 2017. Disponible en:

http://www.repositorio.cedro.org. pe/bitstream/CEDRO/361/3/CED RO.Estudio\%20EPI.2017.pdf

2. Contreras, L., Molina,V. y Cano,C. (2011), Consumo de drogas en adolescentes con conductas infractora: análisis de variables psicosociales implicadas
2011;24(1):31-38.

3. Arangoitia, A. Clima Social Familiar y Agresividad en adolescentes de 3ero, 4to y 5to de secundaria de la Institución Educativa Parroquial San Columbano 2017 Lima, Perú. 5. Vargas H, Tovar H, Valverde J. Universidad César Vallejo. Disponible en: http://repositorio.ucv.edu.pe/bitstr eam/handle/UCV/3617/Arangoitia _PAI.pdf
4. Rivera R, Cuentas MC. Influencia de la familia sobre las conductas antisociales en adolescentes de Arequipa-Perú. Actualidades en Psicología. 2016 Jun 27;30(120):85-97.

Prevalencia y factores asociados con el episodio depresivo en adolescentes de Lima Metropolitana y Callao. Revista Peruana de Epidemiología. 2010;14(2):1-8. 
6. Chirinos Cazorla PL. Violencia escolar y desarrollo de habilidades socioemocionales de estudiantes de secundaria de instituciones educativas de distritos con mayor índice de violencia de Lima Metropolitana. Lima, Perú. Universidad peruana Cateyano Heredia, [Citado 28 enero 2019] Disponible en: http://repositorio.upch.edu.pe/bitst ream/handle/upch/3563/Violencia _ChirinosCazorla_Paula.pdf?sequ ence $=1 \&$ is Allowed $=y$

7. Burga V, Delgado G, Inteligencia emocional en mujeres adolescentes convivientes que asisten a un hospital provincial de Lambayeque - 2014 [Internet] Perú. Universidad Católica Santo Toribio [Citado 28 enero 2019] Disponible en: http://tesis.usat.edu.pe/bitstream/u sat/713/1/TL_BurgaRubio Valery_ DelgadoTorres Gabriela.pdf

8. García-Álvarez D, José-Soler M, Achard-Braga L. Promoción del bienestar psicológico en la secundaria: una experiencia piloto. Búsqueda. 2017 Jun 27;4(18):22-35.

9. Ugarriza N, Pajares-Del-Águila L. La evaluación de la inteligencia emocional a través del inventario de BarOn ICE: NA, en una muestra de niños y adolescentes. Persona. 2005 Nov 4(008):11-58.

10. Vigoa EV. Inteligencia emocional y rendimiento académico en estudiantes de Medicina Humana de la Universidad Nacional de la Amazonía Peruana. Ciencia y Desarrollo. 2010 Dec 1;12:41-56.

11. Espinoza V. Bienestar psicológico y autoeficacia percibida en adolescentes institucionalizados por situación de abandono. Perú
Pontificia Universidad Católica del Perú. [Consultado 27 enero 2019] Disponible en: http://tesis.pucp.edu.pe/repositori o/bitstream/handle/123456789/71 67/ESPINOZA_PAREDES_VIC TOR_BIENESTAR.pdf?sequence $=1$

12. Goleman D. La práctica de la inteligencia emocional. Editorial Kairós;

1999.

13. Salguero JM, Fernández-Berrocal P, Ruiz-Aranda D, Castillo R, Palomera R. Inteligencia emocional y ajuste psicosocial en la adolescencia: El papel de la percepción emocional. European journal of education and psychology. 2015;4(2):143-152.

14. Barahona LM, Alegre AA. Emotional Intelligence and ADHD: A Comparative Analysis in Students of Lima Metropolitan Area. Journal of Educational Psychology-Propósitos

Representaciones. 2016;4(1):89114.

15. Aranguren $\mathrm{M}$, Irrazabal $\mathrm{N}$. Estudio de las propiedades psicométricas de las escalas de bienestar psicológico de Ryff en una muestra de estudiantes argentinos. Ciencias Psicológicas. 2015 May;9(1):73-83.

16. Hernández R. Metodología de investigación. $6^{\circ}$ (ed.). México: Interamericana editores; 2014.

17. Casullo MM, Solano AC. Evaluación del bienestar psicológico en estudiantes adolescentes argentinos. Revista de Psicología. 2000;18(1):35-68.
18. Bermúdez MP, Teva Álvarez I, Sánchez A. Análisis de la relación entre inteligencia emocional, estabilidad emocional y bienestar psicológico. Universitas Psychologica. 2003;2(1):27-32.

19. Delgado-Rodríguez RF. Relaciones interpersonales en la adolescencia: Implementación de un programa de entrenamiento en asertividad y habilidades sociales para adolescentes de $1^{\circ}$ y $2^{\circ}$ de la ESO. España. Universidad de Granada. [Consultado 28 enero 2019] Disponible en: https://www.researchgate.net/p ublication/47440974_Relacion es_interpersonales_en_la_adol escencia_Implementacion_de_ un_programa_de_entrenamient o_en_asertividad_y_habilidade s_sociales_para_adolescentes_ de_1_y_2_de_la_ESO

20. Meneses Gómez MV, Ruiz Sánchez S, Sepúlveda Henao M. Revisión de las principales teorías de bienestar psicológico. Antioquia, Colombia. Universidad de Antioquia [Consultado 28 enero 2019] de: http://200.24.17.74:8080/js pui/bit stream/fcsh/517/3/Meneses Victori a_2016_RevisionPrincipales Teori as BienestarPsicologico.pdf

21. Gómez-Bustamante EM, CogolloMilanés Z. Asociación entre religiosidad y estilo de vida en adolescentes. Revista de la Facultad de Medicina. 2015 Apr 1;63(2):193-8.

22. Pulido F, Herrera F. Predictores de la felicidad y la inteligencia emocional en la educación secundaria. Revista Colombiana de Psicología. 2018;27(1):71-84. 